\title{
Multilyzer - Microfluidic Online Measuring System for the Analysis of Environmentally Relevant Substances

\author{
B. Blahnikova ${ }^{1}$, D. Müller ${ }^{1}$, M. Waas ${ }^{1}$, A. Lechner ${ }^{1}$
}

${ }^{1}$ Centre of Excellence Nanochem, Faculty of General Studies and Microsystems Technology, OTH

Regensburg, Seybothstaße 2, 93053 Regensburg, Germany

alfred.lechner@oth-regensburg.de

\begin{abstract}
:
Excessive use of nitrogen based fertilizers in agriculture represents an environmental hazard. Thus, analysis of soil water and groundwater has become an essential part of substainability and ecological preservation. On this account, the Multilyzer provides a suitable solution.

The Multilyzer is a microfluidic online measuring system for the determination of lowest concentrations of nitrate, nitrite and ammonium, all of which are essential components of the nitrogen cycle. The transportable system allows an online measurement and is driven by a 12 Volts battery. Consequently, conversion and degradation processes can be avoided, unlike the analysis in a local laboratory. The measurement is based on absorption and fluorescence spectroscopy. The complete device is developed at the Centre of Excellence Nanochem at OTH Regensburg including fabrication technologies, software and hardware controlling.
\end{abstract}

Key words: spectroscopy, environment, nitrate, microfluidics, online measurement

\section{Introduction}

The focus of our research is laid on the measurement of contamination in soil water, considering ammonium $\left(\mathrm{NH}_{4}^{+}\right)$, nitrite $\left(\mathrm{NO}_{2}{ }^{-}\right)$, nitrate $\left(\mathrm{NO}_{3}{ }^{-}\right)$which are the principle components of the nitrogen cycle. Common analysis methods enable the detection of lowest concentrations of the above-mentioned substances. However, they do not exactly meet the demands of the customer, as far as usability and flexibility are concerned. The main specifications the research is based on are:

- Measurement precision in the lowest ppm range (parts per million)

- Real-time analysis

- Manageable size and cost-efficiency must be considered as important aspects.

- Low sensitivity of the measurement system towards external influences

To comply with these specifications, we intend to deliver an environmental monitoring solution by means of microtechnological processing. The Multilyzer [1] measurement system consists of a silicon based measurement cell and peripheral equipment to operate the cell. The specimen is analysed via absorption and emission (fluorescence) spectroscopy. The technologies are based on projects like Picolyzer [2], [3].

\section{Design and Methodology}

The Multilyzer device consists of several components (see Fig. 1). A silicon based measurement cell with four fluidic channels is used for measuring samples. The channels are screened by LEDs (one LED per channel) through optical fiber connections, and the signals are captured by photodiodes (absorption) or a spectrometer (fluorescence). A microcontroller regulates the stabilisation of the LEDs for a continuous spectrum and collects the measured data of the photodiodes. It also controls the micropump unit to pump the measured samples into the channels. A Raspberry Pi with internally developed software gathers data from the microcontroller and the spectrometer for calculation and visualisation of the measured data (graphs) and allows to change settings on the microcontroller.

\section{A. Measurement Cell}

As the core of the Multilyzer system the measurement cell is fabricated in the clean room at the OTH Regensburg. All applied microtechnological processes are well known and easily reproducible. The manufacturing of a silicon and glass based measurement cell is carried out in 9 steps (see Fig. 2). 


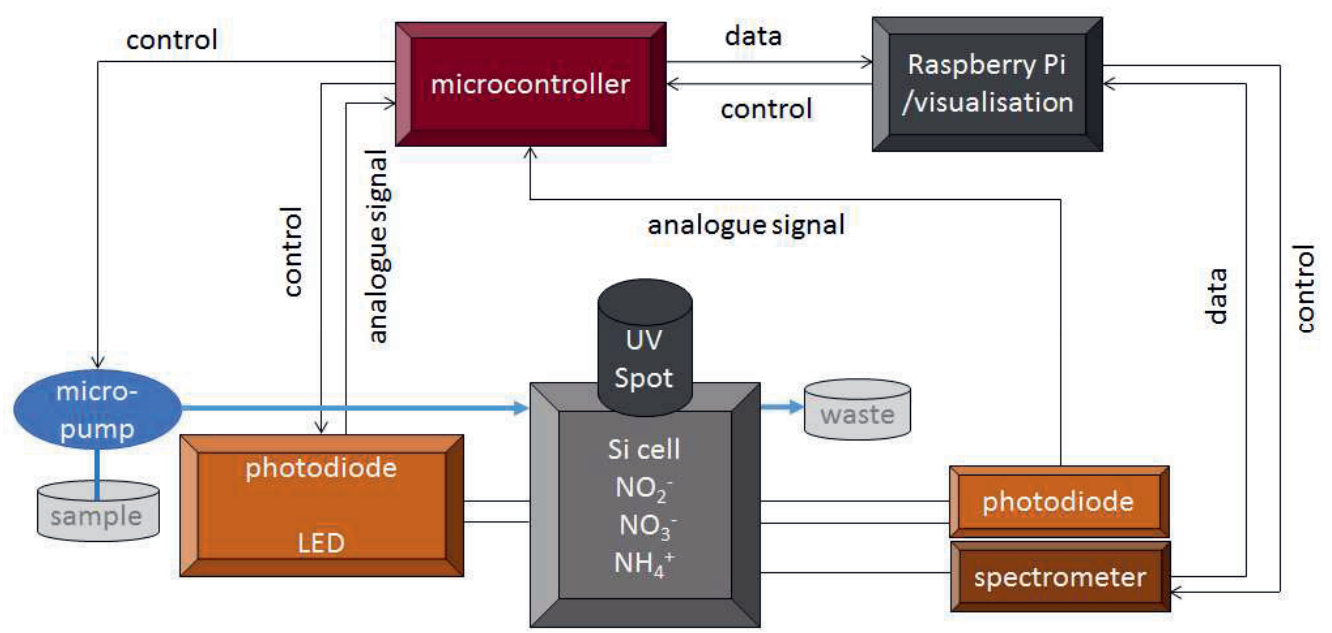

Fig. 1. Schematic layout of the Multilyzer system.


Fig. 2. Fabrication process of a silicon and glass based Multilyzer measurement cell.

Initially, the seven-inch photomask is designed. The pattern contains four microfluidic channels, each one of them 280 micrometres wide. There are two longer channels for the absorption measurement of lower analyte concentrations and two short channels for high analyte concentrations and fluorescence measurement. All channels are designed with two curved fluid confluxes for sample introduction. Structuring of the six-inch silicon wafer via Photolithography starts with spin coating of the positive photoresist. After UV light exposure the photoresist is developed in diluted tetramethylammonium hydroxide (TMAH).
Subsequently, the wafer is etched via Reactive Ion Etching. Deep Reactive Ion Etching (DRIE) alternates etching into the silicon substrate with sulphur hexafluoride $\left(\mathrm{SF}_{6}\right)$ and passivation of the etched structure with fluoroform $\left(\mathrm{CHF}_{3}\right)$. Depending on the etch rate, the two steps are repeated approximately 200 times, generating deep etch pits with a high aspect ratio. After removal of the photoresist with Caro's acid $\left(\mathrm{H}_{2} \mathrm{O}_{2} / \mathrm{H}_{2} \mathrm{SO}_{4}\right)$ the substrate is covered with borosilicate glass via anodic bonding. LED light is launched into the measurement channel via fibre optic cables which are inserted into the channels and fixed with two-component 
adhesive. For fluorescence measurement, an UV spot fixture is placed above one of the short measuring section and nanoports are installed at the fluid conflux. For sample introduction capillary tubes are plugged in the nanoports.

To protect the cell from environmental influences and fix the individual elements the completed cell is encapsulated with plastic compound.

\section{B. Measurement and Control}

To transmit light through the measurement cell it's essential to couple the LED light sources with the optic fibres. An optical unit is designed by $C A D$ and produced by $3 D$ printing. The main function is coupling the light into the fibres. It also contains an optical filter to lower wavelength shifts caused by self-heating or ambient temperature changes. In addition, a photodiode is positioned in the unit to measure the reference intensity for normalisation (see Fig. 3)

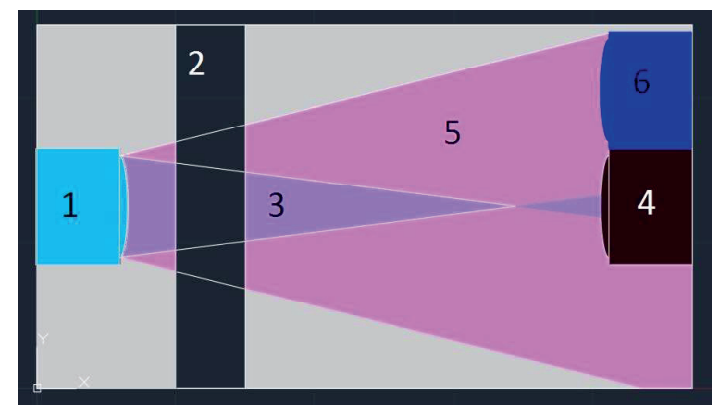

Fig. 3. Scematic of the optical Unit. UV- LED light source [1] ist filtered by an optical bandpass [2]. Due to the lens on the led, there is a Main Beam [3] coupled through a collimator lens [4] into fibreoptics and a scattered Beam [5] used for measuring the LED output by a Photodiode [6].

A microcontroller is used to connect analogue sensors to the digital computing system. As well as controlling the LED light sources by PIcontrol (proportional integral control). The analogue signals are detected by A/D converters, digitalized and sent via serial communication to the Raspberry $\mathrm{Pi}$. To control and measure the brightness of the LED's we use photodiodes. For compensation of temperature shifts the use of similar photodiodes is necessary. The resulting advantage is that the ratio of transmitted light intensity and reference intensity (Itrans//ref) remains constant if only the temperature changes.

Furthermore, the use of a temperature sensor enables the microcontroller to compensate temperature shifts of the whole system. The microcontroller also handles a micropump connected by a micropump driver IC (integrated circuit).

To increase the flexibility of the system it's possible to set or change parameters like LED intensity or pump frequency during runtime.

\section{Data Evaluation and Visualisation}

The Raspberry $\mathrm{Pi}$ is a waypoint between the Multilyzer cell and the measurement tools, like the spectrometer (fluorescence) and the microcontroller (absorption). It enables the calculation and visualization of the real time and long term measurement on a touchscreen display and allows to save long term measurement results for analysing purposes.

On the Graphical User Interface of the Raspberry $\mathrm{Pi}$ the user can initiate and stop a measurement. Over a serial connection to the microcontroller settings get transferred to control the micropumps and UV-LEDs. The microcontroller returns raw data to the Raspberry Pi to calculate and draw the graphs afterwards.

The Graphical User Interface is realized with the Qt-Framework and drawing the graphs with the open source library qwt. The utilisation of a Raspberry $\mathrm{Pi}$ enables to communicate in several kinds of transferring and saving the online long term measurement.

There are two principles for calculating the intensities of light, absorption and fluorescence. Absorption is based on the Beer-Lambert law which is defined as in eq. (1).

$$
E=c \cdot \varepsilon \cdot d=\log \left(\frac{I_{0}}{I}\right)
$$

$E$ is the extinction, $c$ the concentration of the sample, $d$ the measurement channel length, lo the intensity of incident light and I the intensity of weakened light.

Fluorescence light intensity $I_{f}$ is calculated as in eq. (2).

$$
\text { If }=2.303 \cdot \Phi \cdot I_{0} \cdot \varepsilon \cdot c \cdot x
$$

$\mathrm{l}_{0}$ is the incident light intensity $\varepsilon$ extinction coefficient, $\Phi$ the quantum efficiency and $x$ is the path length. The factor 2.303 results from the conversion of decadic logarithm to natural logarithm. 


\section{Results}

The result of the fabrication process is a fully functioning measurement cell (see Fig. 4).

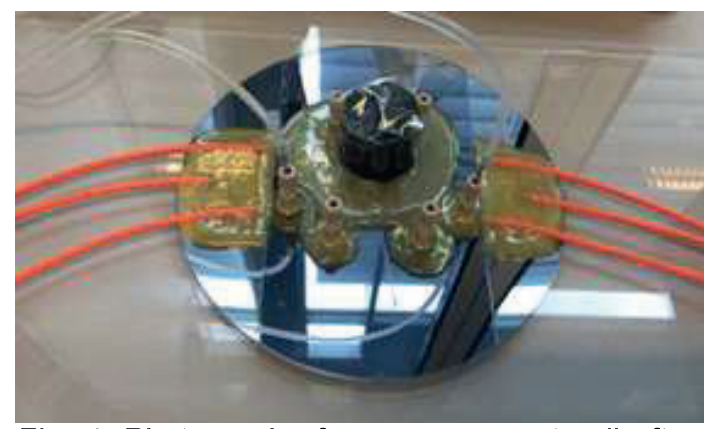

Fig. 4. Photograph of a measurement cell after anodic bonding and the application of the required components for measurement, eg. fibre optic cables (orange), UV spot fixture (black) and nanoports with tubing. In this case three channels are available.

For the inclusion of the cell in a transportable Multilyzer system the cell is encapsulated in casting compound (see Fig. 5).

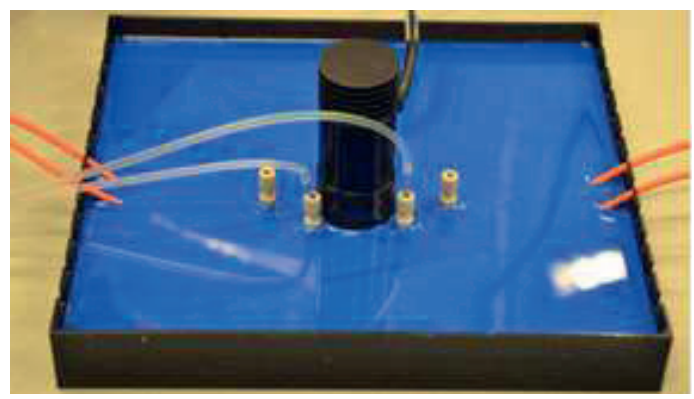

Fig. 5. Photograph of a measurement cell after covering with a blue casting compound. In this cell two of four channels, one 6.6 centimetres long and one 3.3 centimetres long, were chosen for measurement.

A step measurement of nitrate is shown in Fig. 6. Nitrate and nitrite concentrations down to 10 ppm can be determined.

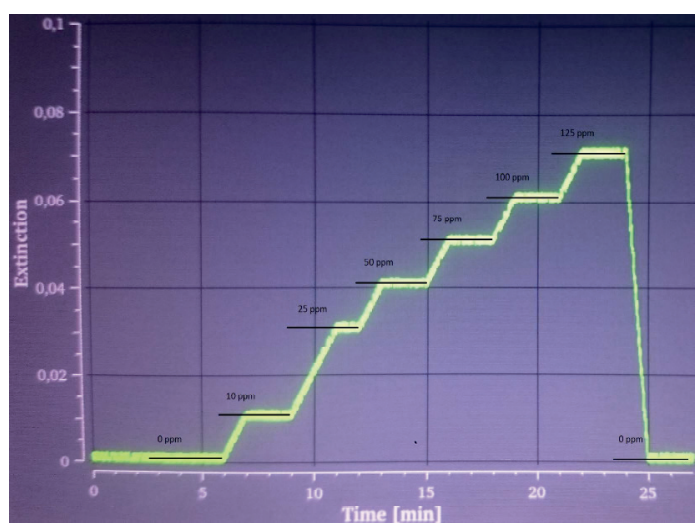

Fig. 1. Ideal step measurement of different nitrate concentrations as displayed on the user interface of the Raspberry Pi.

\section{Conclusion}

The Multilyzer system is a suitable solution for nitrate measurement in fluid samples. As it is transportable, user friendly, economical and guarantees the measurement of lowest analyte concentrations it meets the required specifications. Further research will be focussed on the development of stable and precise ammonium measurement. Moreover, the system must be prepared for long time field measurement. This includes the design of a weather-resistant enclosure and a 12 Volts storage battery.

\section{References}

[1] Blahnikova, A. Lechner Multilyzer - Microfluidic Online Measuring System for the Analysis of Environmentally Relevant Substances; . Applied Research Conference 2016 - Amberg, pages 63 68. Pro BUSINESS GmbH, 1st Edition, 2016.

[2] M. Bauhuber, A. Mikrievskij, A. Lechner: Isotropic wet chemical etching of deep channels with optical quality in silicon with HNA based etching solutions, Material Science in Semiconductor Processing, Vol. 16, pp. 1428-1433, 2013.

[3] A. Hutterer, M. Bauhuber, H. Hummel, A. Lechner: Real-time analyses of metal contaminations in the ppb-range; Solid State Phenomena Vol. 195 (2013), pp 269-273; Trans Tech Publications, Switzerland, 2013. 